\title{
Dynamic range of nanotube- and nanowire-based electromechanical systems
}

\author{
H. W. Ch. Postma, I. Kozinsky, A. Husain, and M. L. Roukes ${ }^{\text {a) }}$ \\ Condensed Matter Physics, California Institute of Technology 114-36, Pasadena, California 91125
}

(Received 17 January 2005; accepted 1 April 2005; published online 25 May 2005)

\begin{abstract}
Nanomechanical resonators with high aspect ratio, such as nanotubes and nanowires are of interest due to their expected high sensitivity. However, a strongly nonlinear response combined with a high thermomechanical noise level limits the useful linear dynamic range of this type of device. We derive the equations governing this behavior and find a strong dependence $\left[\propto d \sqrt{(d / L)^{5}}\right]$ of the dynamic range on aspect ratio. (C) 2005 American Institute of Physics.
\end{abstract}

[DOI: 10.1063/1.1929098]

The limits of mechanical detection with nanoelectromechanical systems (NEMS) are being actively pursued for sensing applications, such as the attainment of sub-attonewton force sensing for magnetic resonance force microscopy, ${ }^{1}$ sub-attogram mass sensing, ${ }^{2,3}$ mechanical single spin detection, ${ }^{4}$ or the study of mechanical motion approaching the quantum regime. ${ }^{5-8}$ Applications like these require both high responsivity and ultra-high-frequency operation. ${ }^{9}$ Both can be attained simultaneously with small diameter, large aspect ratio doubly clamped resonators. Nanoscale materials such as carbon nanotubes or nanowires are a natural choice for these resonators due to their intrinsic small size. We recently reported a bottom-up nanomechanical resonator, a Pt nanowire, and found that it takes a very low driving power to bring this device into the nonlinear regime. ${ }^{10}$ Here, we show how the onset of this nonlinear regime decreases with decreasing diameter, while the thermomechanical noise increases with aspect ratio. We conclude that the useful linear dynamic range of such devices is severely limited, with the result that many applications will involve operation in the nonlinear regime.

A typical layout for a doubly clamped nanomechanical resonator is shown in Fig. 1. The resonator can be driven and detected in several ways, e.g., magnetomotively, ${ }^{11}$ or optically. ${ }^{12}$ The driving force $f(t)$ leads to a time dependent bending profile $z(x, t)$, which can be found by solving the differential equation

$$
\begin{aligned}
\mathcal{L}[z(x, t)]= & E I z_{x x x x}-\left(T_{0}+\frac{E A}{2 L} \int_{0}^{L} z_{x}^{2} \mathrm{~d} x\right) z_{x x} \\
& +\rho A z_{t t}-f(z, t)=0
\end{aligned}
$$

with boundary conditions $z(0)=z(L)=z_{x}(0)=z_{x}(L)=0$. Here, $A$ is the cross-sectional area, $E$ is Young's modulus, $\rho$ is the density, and $I$ is the moment of inertia about the longitudinal axis of the beam. The term in between brackets describes tension in the beam, and is a sum of residual tension $T_{0}$ and a bending-induced tension, respectively.

Since Eq. (1) cannot be solved exactly we use the Galerkin discretization procedure, ${ }^{13}$ representing the solution to Eq. (1) in terms of a linearly independent set of basis functions $\phi_{n}(x)$ where each basis function satisfies the boundary

\footnotetext{
${ }^{\text {a) }}$ Author to whom correspondence should be addressed; electronic mail:
} roukes@caltech.edu conditions. The error associated with this approximation technique is

$$
e=\mathcal{L}\left[\sum_{n=1}^{N} z_{n}(t) \phi_{n}(x)\right]-\mathcal{L}[z(x, t)] .
$$

The Galerkin procedure requires this error to be orthogonal to each basis function, or in other words, the error is a residual that cannot be expressed in terms of the given finite set of basis functions:

$$
\int_{0}^{L} e \phi_{n}(x) \mathrm{d} x=0 \text {. }
$$

We are interested in the response of the beam at resonance when the first mode is dominant, so it suffices to consider the case $n=1$. For a doubly clamped beam, the simplest function that approximates the first mode is $\phi_{1}(x)=\sqrt{2 / 3}[1$ $-\cos (2 \pi x / L)] .{ }^{14,15}$ The normalization $\sqrt{2 / 3}$ ensures that the time dependent amplitude $z_{1}(t)$ we find is the root mean square displacement averaged over the length of the beam (not time).

Performing the integration and noticing that $\mathcal{L}[z(x, t)]$ $=0$ as given in the Eq. (1), we arrive at the Duffing-type equation describing the time-varying behavior of the system:

$$
\ddot{z}_{1}(t)+\omega_{0}^{2} z_{1}(t)+\alpha z_{1}^{3}(t)=0
$$

with

$$
\omega_{0}=\frac{4 \pi^{2}}{L^{2}} \sqrt{\frac{E I}{3 \rho A}\left(1+\frac{L^{2} T_{0}}{4 \pi^{2} E I}\right)}, \quad \alpha=\frac{E}{18 \rho}\left(\frac{2 \pi}{L}\right)^{4}
$$

for free vibrations of the elastic beam $\{f[z(x), t] \equiv 0\}$. The resonant frequency $\omega_{0}$ obtained in this approximation is slightly higher than the exact value. We add a phenomeno-

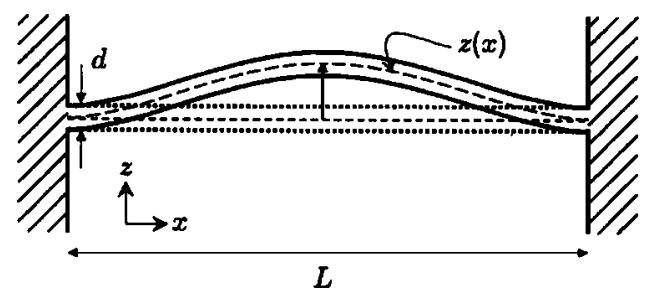

FIG. 1. Schematic representation of a doubly clamped nanomechanical resonator of length $L$ and diameter $d$. An applied force leads to a bending profile $z(x)$ as indicated. 


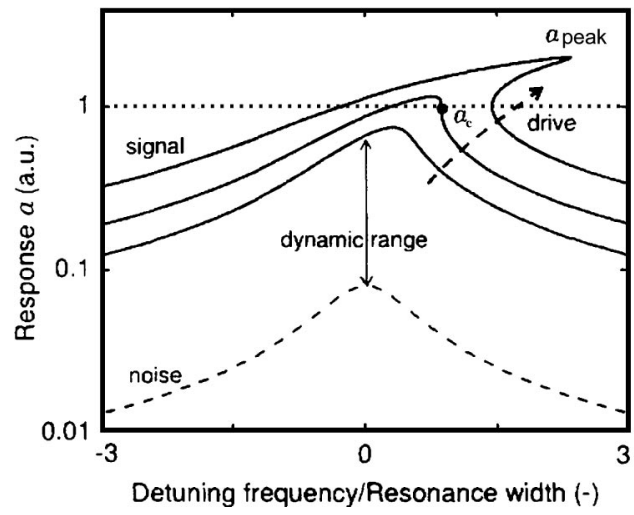

FIG. 2. Solid lines indicate typical response of a Duffing oscillator as a function of frequency with increasing driving strength. The solid lines are plots of equation $2 Q\left(\omega-\omega_{0}\right) / \omega_{0}=\frac{2}{3} \sqrt{3} a^{2} / a_{c}^{2} \pm \sqrt{a_{\text {peak }}^{2} / a^{2}-1}$, where $a_{c}$ is chosen 1 , for $a_{\text {peak }}=0.745,2 / \sqrt{3}$, 2. (Ref. 13). The dashed line is a plot for $a_{\text {peak }}=0.1$, indicating an arbitrarily chosen rms noise floor.

logical damping term $\dot{z}_{1} \omega_{0} / Q$ to Eq. (4), where $Q$ is the mechanical quality factor as obtained in the linear regime. Then the critical amplitude for the onset of nonlinearity is ${ }^{13}$

$$
a_{c}=\omega_{0} \frac{L^{2}}{\pi^{2}} \sqrt{\frac{\rho \sqrt{3}}{E Q}} .
$$

The critical amplitude describes at what displacement nonlinearity sets in: a smaller value of $a_{c}$ signifies an earlier onset of nonlinearity and generally a stronger nonlinear behavior. A nanotube or a nanowire can be well described by a cylindrical rod with diameter $d: A=\pi d^{2} / 4$ and $I=\pi d^{4} / 64$. We parametrize a rectangular beam with width $d$ in the direction of motion and thickness $t$, yielding $A=d t$ and $I$ $=t d^{3} / 12$. This gives us

$$
\begin{aligned}
& a_{c}=\frac{2}{\sqrt[4]{3}} \sqrt{\frac{1}{Q}\left(\frac{d^{2}}{4}+\frac{4 T_{0}}{\pi^{3} E} \frac{L^{2}}{d^{2}}\right)} \quad \text { (cylindrical) } \\
& a_{c}=\frac{2}{\sqrt[4]{3}} \sqrt{\frac{1}{Q}\left(\frac{d^{2}}{3}+\frac{T_{0}}{\pi^{2} E} \frac{L^{2}}{t d}\right)} \quad \text { (rectangular) }
\end{aligned}
$$

A typical response of a Duffing oscillator described by Eq. (4) is shown in Fig. 2. At low driving strength, the amplitude has the Lorentzian shape from the linear regime. Increasing the driving strength causes the peak to be pulled over to high frequencies at high amplitudes. A common definition of the onset of nonlinearity is the $1 \mathrm{~dB}$ compression point, i.e., the point at which the signal is $1 \mathrm{~dB}$ lower than expected for the case of purely linear response. At resonance, this happens when $a_{\text {peak }}=0.745 a_{c}$, which is the lowest solid curve in Fig. 2. This sets the upper limit of the useful linear range.

The lower limit of the dynamic range is set by the incoherent sum of all stochastic processes driving the resonator, ${ }^{16}$ such as thermomechanical fluctuations, quantum noise, noise from adsorption and desorption of gaseous species, ${ }^{17}$ and extrinsic sources such as vibrational and instrumental (readout) noise. For simplicity, and in the spirit of considering ultimate thermodynamic limits, we solely consider thermomechanical noise. The spectral density of displacement noise on resonance is

Downloaded 14 Dec 2005 to 131.215.225.171. Redistribution subject to AIP license or copyright, see http://apl.aip.org/apl/copyright.jsp

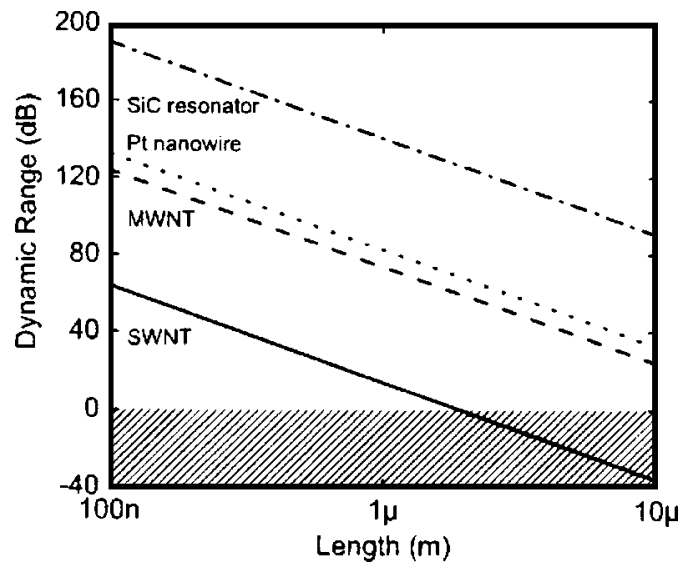

FIG. 3. Dynamic range at $4 \mathrm{~K}$ for several doubly clamped resonators as indicated in the figure and Table I. The shaded region below $0 \mathrm{~dB}$ indicates the absence of a linear region of operation.

$$
S_{x}=\frac{4 k_{B} T Q}{m \omega_{0}^{3}},
$$

where $m$ is the total resonator mass, $m=\pi \rho L d^{2} / 4$.

We now define the useful dynamic range (DR) as the ratio of the $1 \mathrm{~dB}$ compression point $\left(0.745 a_{c}\right)$ to the noise amplitude at resonance

$$
\mathrm{DR}(\mathrm{dB}) \equiv 20 \log \left(\frac{0.745 a_{c}}{\sqrt{2 S_{x} \Delta f}}\right),
$$

where $\Delta f$ is the measurement bandwidth $(\Delta f=1$ in Fig. 3), and the $\sqrt{2}$ comes from the conversion of $a_{c}$ to rms.

For the moment neglecting the residual tension $T_{0}$,

$$
\begin{aligned}
& \mathrm{DR}=20 \log \left[2.41 d\left(\frac{d}{L}\right)^{5 / 2}\right. \\
& \left.\times \sqrt{\frac{E^{3 / 2}}{Q^{2} k_{B} T \Delta f \sqrt{\rho}}}\right] \quad \text { (cylindrical) } \\
& \mathrm{DR}=20 \log \left[3.9 \sqrt{d t}\left(\frac{d}{L}\right)^{5 / 2}\right. \\
& \left.\times \sqrt{\frac{E^{3 / 2}}{Q^{2} k_{B} T \Delta f \sqrt{\rho}}}\right] \quad \text { (rectangular). }
\end{aligned}
$$

It is apparent that the dynamic range depends strongly on the aspect ratio $L / d$ and directly on diameter. Figure 3 shows the dynamic range plotted for several device geometries of interest: a single-wall carbon nanotube (SWNT), a multiwall carbon nanotube (MWNT), a Pt nanowire, ${ }^{10}$ and a SiC rectangular beam ${ }^{18}$ with parameters as given in Table I. We assume a mechanical quality factor $Q$ for MWNT and SWNT of about 1000, although room temperature measurements ${ }^{19}$ indicate a lower value. The dynamic range is very limited, and

TABLE I. Input parameters for Fig. 3.

\begin{tabular}{lcccc}
\hline \hline & $d(\mathrm{~nm})$ & $\rho\left(\mathrm{kg} / \mathrm{m}^{3}\right)$ & $E(\mathrm{TPa})$ & $Q$ \\
\hline SWNT & 1.4 & 1930 & 1 & 1000 \\
MWNT & 20 & 1930 & 1 & 1000 \\
Pt nanowire (Ref. 10) & 43 & 21060 & 0.168 & 8500 \\
SiC beam (Ref. 18) & $150(t=100)$ & 2880 & 0.430 & 8000 \\
\hline \hline
\end{tabular}


in the case of SWNTs even drops below $0 \mathrm{~dB}$ above a length of $2 \mu \mathrm{m}$, which renders the device useless as a linear detector. It is worth nothing that a change in temperature or measurement bandwidth shifts these curves along the vertical axis, but does not change the scaling behavior. Generally, the resonator with the smallest diameter will have the smallest dynamic range.

We now discuss the effect of residual tension $T_{0}$. It may arise from differential thermal contraction, ${ }^{10}$ or may be induced by a dc voltage on a gate nearby. ${ }^{20}$ Increasing tension will increase the resonant frequency. We therefore account for the presence of tension by using $a_{c}$ from Eq. (5);

$$
\mathrm{DR}=20 \log \left(3.08 \frac{\left(f_{0} L\right)^{5 / 2} d \rho}{Q \sqrt{E k_{B} T \Delta f}}\right),
$$

where $\omega_{0} \equiv 2 \pi f_{0}$. The dynamic range can therefore be increased or decreased through a change in resonant frequency due to tension. An upper limit to the frequency tuning is set by how high tension can be before deforming, breaking, or irreversibly pulling the resonator to the gate. ${ }^{21}$ This places an upper limit on the available dynamic range, but that limit is strongly dependent on the actual sample geometry, tuning mechanism, and material properties, and is therefore not treated here.

It has been observed that the mechanical quality factor $Q$ also depends on the dimensions of the resonator, which would alter the scaling behavior presented in this letter. For instance, in an empirical study, it was shown that $Q$ scales roughly as the inverse of the surface to volume ratio. ${ }^{22}$ In another study, it was shown that extra dissipation may also arise from eddy current damping ${ }^{11}$ which scales as $\left(Q^{-1}\right.$ $\left.-Q_{0}^{-1}\right) \propto(L / d)^{3}$, where $Q_{0}$ is the quality factor without eddy current damping. Many other dissipation mechanisms exist, and it is therefore difficult to derive a general scaling law of $Q$ with sample parameters.

Finally, we note that for singly clamped cantilevers, the nonlinearity generally sets in at a larger amplitude than in the doubly clamped beams we discuss. This is due to different sources of nonlinearity in cantilevers: the nonlinearity due to curvature rather than tension dominates for the fundamental mode of the cantilever. ${ }^{23}$ If nanowires and nanotubes are used in AFM-type tapping mode, nonlinearities in tipsurface interaction become important as well. ${ }^{24}$

We have shown that for large aspect ratio resonators, one is forced to work close to the nonlinear regime or even in it, a rather undesirable situation for using nanoresonators as linear sensors. This new nonlinear regime that promises to dominate the nanoscale beyond the conventional dynamic range, however, might offer as yet unexplored opportunities for noise reduction and signal enhancement in nanoresonators. Charge detection is one such application. Charge can be detected at a fraction of a single electron in the mechanical domain, ${ }^{6,25}$ since the presence of charge on a gate nearby shifts the resonant frequency. By operating the resonator at the onset of nonlinearity close to the infinite negative slope $\mathrm{d} A / \mathrm{d} \omega$ at $a_{c}$ in Fig. 2, the charge sensitivity can be increased drastically, as suggested by Krömmer et al. ${ }^{26}$ Another application of nonlinear mechanics is to use the nonlinear resonator as the frequency stabilizing element in a feedback loop. It has been shown that the long term phase stability of such an oscillator can be improved considerably with this technique. $^{27}$

The authors acknowledge support from DARPA/MTO (SPAWAR) under Grant No. N66001-02-1-8914. We thank I. Bargatin, E. Myers, O. Kogan, X. L. Feng, and M. C. Cross for useful discussions.

${ }^{1}$ J. A. Sidles, J. L. Garbini, K. J. Bruland, D. Rugar, O. Zuger, S. Hoen, and C. S. Yannoni, Rev. Mod. Phys. 67, 249 (1995).

${ }^{2}$ M. L. Roukes and K. L. Ekinci, U.S. Provisional Patent Application No. 60/288,741 (2001), and U.S. Patent No. 6,722,200 (2004).

${ }^{3}$ K. L. Ekinci, X. M. H. Huang, and M. L. Roukes, Appl. Phys. Lett. 84, 4469 (2004).

${ }^{4}$ D. Rugar, R. Budakian, H. J. Mamin, and B. Chui, Nature (London) 430, 329 (2004).

${ }^{5}$ A. D. Armour, M. P. Blencowe, and K. C. Schwab, Phys. Rev. Lett. 88, 148301 (2002).

${ }^{6}$ R. G. Knobel and A. N. Cleland, Nature (London) 424, 291 (2003).

${ }^{7}$ M. D. LaHaye, O. Buu, B. Camarota, and K. C. Schwab, Science 304, 74 (2004).

${ }^{8}$ A. Cho, Science 299, 36 (2003).

${ }^{9}$ X. M. H. Huang, C. A. Zorman, M. Mehregany, and M. L. Roukes, Nature (London) 421, 496 (2003).

${ }^{10}$ A. Husain, J. Hone, H. W. C. Postma, X. M. H. Huang, T. Drake, M. Barbic, A. Scherer, and M. L. Roukes, Appl. Phys. Lett. 70, 1327 (2003).

${ }^{11}$ A. N. Cleland and M. L. Roukes, Sens. Actuators, A 72, 256 (1999).

${ }^{12}$ D. W. Carr, S. Evoy, L. Sekaric, H. G. Craighead, and J. M. Parpia, Appl. Phys. Lett. 75, 920 (1999).

${ }^{13}$ A. H. Nayfeh and D. T. Mook, Nonlinear Oscillations, Physics and Applied Mathematics: A Wiley-Interscience Series of Texts, 1st ed., Monographs and Tracts (John Wiley, New York, 1979).

${ }^{14}$ J. G. Eisley, J. Appl. Math. Phys. 15, 167 (1964).

${ }^{15}$ W.-Y. Tseng and J. Dugundji, J. Appl. Mech. 37, 292 (1970).

${ }^{16}$ A. Cleland and M. L. Roukes, J. Appl. Phys. 92, 2758 (2002).

${ }^{17}$ K. L. Ekinci, Y. T. Yang, and M. L. Roukes, J. Appl. Phys. 95, 2682 (2004).

${ }^{18}$ X. M. H. Huang, M. K. Prakash, C. A. Zorman, M. Mehregany, and M. Roukes, in The 12th International Conference on Solid State Sensors,

Actuators, and Microsystems (Transducers '03) (Boston, MA, 2003).

${ }^{19}$ V. Sazonova, Y. Yaish, H. Ustunel, D. Roundy, T. A. Arias, and P. L. McEuen, Nature (London) 431, 284 (2004).

${ }^{20}$ S. Sapmaz, Y. M. Blanter, L. Gurevich, and H. S. J. van der Zant, Phys. Rev. B 67, 235414 (2003).

${ }^{21}$ M. Dequesnes, S. V. Rotkin, and N. R. Aluru, Nanotechnology 13, 120 (2002).

${ }^{22}$ P. Mohanty, D. A. Harrington, K. L. Ekinci, Y. T. Yang, M. J. Murphy, and M. L. Roukes, Phys. Rev. B 66, 085416 (2002).

${ }^{23}$ T. Anderson, A. H. Nayfeh, and B. Balachandran, J. Vibr. Acoust. 118, 21 (1996).

${ }^{24}$ S. I. Lee, S. W. Howell, A. Raman, and R. Reifenberger, Phys. Rev. B 66, 115409 (2002).

${ }^{25}$ A. Cleland and M. Roukes, Nature (London) 392, 160 (1998).

${ }^{26}$ H. Krömmer, A. Erbe, A. Tilke, S. Manus, and R. Blick, Europhys. Lett. 50, 101 (2000).

${ }^{27}$ D. S. Greywall, B. Yurke, P. A. Busch, A. N. Pargellis, and R. L. Willett, Phys. Rev. Lett. 72, 2992 (1994). 